\title{
Evolution of the YABBY gene family in seed plants
}

\author{
Running Head: YABBY genes in seed plants
}

(Authors)

Cédric Finet ${ }^{1,6 \dagger}$, Sandra K. Floyd ${ }^{2 \dagger}$, Stephanie J. Conway ${ }^{3}$, Bojian Zhong ${ }^{4}$, Charles P. Scutt $^{1}$, John L. Bowman ${ }^{2,5}$

${ }^{1}$ Université de Lyon, Laboratoire de Reproduction et Développement des Plantes, UMR 5667, CNRS, INRA, Université Claude Bernard Lyon I, Ecole Normale Supérieure de Lyon, 46 allée d'Italie 69364 Lyon Cedex 07, France

2 School of Biological Sciences, Monash University, Melbourne Victoria 3800, Australia

${ }^{3}$ School of Botany, University of Melbourne, Melbourne, Victoria 3010, Australia

${ }^{4}$ College of Life Sciences, Nanjing Normal University, Nanjing, China

${ }^{5}$ University of California, Davis, Section of Plant Biology, One Shields Ave., Davis, CA 95616, USA

${ }^{6}$ Current address: Department of Physiology, Development and Neuroscience, University of Cambridge, Downing Street, Cambridge CB2 3DY, UK

${ }^{\dagger}$ These authors contributed equally to this work.

* Two corresponding authors:

Cédric Finet: cedric.finet@ens-lyon.org

John L. Bowman: john.bowman@monash.edu

This is the author manuscript accepted for publication and has undergone full peer review but has not been through the copyediting, typesetting, pagination and proofreading process, which may lead to differences between this version and the Version of Record. Please cite this article as doi: 10.1111/ede.12173 


\begin{abstract}
Members of the YABBY gene family of transcription factors in angiosperms have been shown to be involved in the initiation outgrowth of the lamina, the maintenance of polarity, and establishment of the leaf margin. Whereas most of the dorsal-ventral polarity genes in seed plant have homologs in non-spermatophyte lineages, the presence of YABBY genes is restricted to seed plants. To gain insight into the origin and diversification of this gene family, we reconstructed the evolutionary history of YABBY gene lineages in seed plants. Our findings suggest that either one or two YABBY genes were present in the last common ancestor of extant seed plants. We also examined the expression of YABBY genes in the gymnosperms Ephedra distachya (Gnetales), Ginkgo biloba (Ginkgoales), and Pseudotsuga menziesii (Coniferales). Our data indicate that some YABBY genes are expressed in a polar (abaxial) manner in leaves and female cones in gymnosperms. We propose that YABBY genes already acted as polarity genes in the last common ancestor of extant seed plants.
\end{abstract}

\title{
Introduction
}

The YABBY gene family encodes a class of transcription factors that are specific to seed plants. In angiosperms its members play a pivotal role in lamina development in leaves and leaf-derived organs, e.g., cotyledons, floral organs, outer ovule integuments. The Arabidopsis genome contains six YABBY genes (Sawa et al., 1999)(Siegfried et al., 1999)(Bowman and Smyth, 1999)(Bowman, 2000). Four of these (FILAMENTOUS FLOWER (FIL), YAB2, YAB3, and YAB5) are expressed in leaves and most leaf-derived organs - cotyledons, sepals, petals, stamens and carpels -, whereas expression of CRABS CLAW (CRC) and INNER NO OUTER (INO) are restricted to specific floral organs that are evolutionarily derived from leaves. In contrast to angiosperm YABBY genes, very little is known about the gene content and the putative role of YABBY genes in gymnosperms

YABBY genes encode small proteins composed of an N-terminal zinc finger domain and a C-terminal helix-loop-helix motif (the so-called YABBY domain) similar to a high mobility group box (Bowman and Smyth, 1999). Phylogenetic studies resolve 
angiosperm YABBY genes into five subfamilies, represented in Arabidopsis by FIL + YAB3, CRC, YAB2, YAB5 and INO (Yamada et al., 2004)(Lee et al., 2005)(Yamada et al., 2011). Each of these five subfamilies includes early-diverging ANA-grade angiosperm sequences, suggesting that at least five YABBY genes were present in the last common ancestor of extant flowering plants (Yamada et al., 2011)(Bartholmes et al., 2012). Nevertheless, the relationships between those five subfamilies remain enigmatic due to the lack of an appropriate ougroup.

In Arabidopsis, the vegetative YABBY genes are abaxially expressed in lateral organs and have redundant functions in activating laminar programs and repressing shoot apical meristem programs (Sawa et al., 1999)(Siegfried et al., 1999)(Eshed et al., 2004)(Sarojam et al., 2010). Evidence of similar roles for vegetative YABBY genes has been functionally demonstrated in core eudicots (Golz et al., 2004) and monocots (Juarez et al., 2004a)(Juarez et al., 2004b). In Arabidopsis, CRC is expressed abaxially in the valves and in placental tissues where it functions to specify carpel identity and establish carpel polarity, in addition to being required for proper nectary development (Bowman and Smyth, 1999)(Baum et al., 2001). Expression and functional analyses suggest that $C R C$ acts as a regulator of carpel development across angiosperms (Yamaguchi et al., 2004)(Fourquin et al., 2005)(Orashakova et al., 2009) and nectaries in the core eudicots (Lee et al., 2005). Similarly, Arabidopsis INO expression is restricted to the outermost cell layer of the outer integument of the ovule and promotes outer integument growth (Villanueva et al., 1999). Abaxial expression has been also reported for INO orthologues in eudicots (McAbee et al., 2005), eumagnoliids (Lora et al., 2011), and several representatives of the ANA grade angiosperms (Yamada et al., 2003)(Yamada et al., 2011).

Previous searches of the complete genomes of land plants failed to identify YABBY genes in mosses and lycophytes, suggesting that the YABBY family found in flowering plants can be traced back only to the origin of seed plants (Floyd and Bowman, 2007)(Rensing et al., 2008)(Banks et al., 2011). While YABBY gene function has been investigated extensively in angiosperms, the same is not true of gymnosperm YABBY genes due to the paucity of model systems. To begin to address this question, we compiled a dataset of YABBY sequences across gymnosperms from 
available genomic resources and by experimental cloning. We used the sequences obtained to reconstruct the evolutionary history of seed plant YABBY gene lineages, and also investigated the expression of selected YABBY genes in gymnosperms.

\section{Materials and Methods}

\section{Plant material}

Material of Ephedra distachya L. and Gnetum gnemon L. was collected from plants cultivated in the Botanical Garden 'Parc de la Tête d'Or', Lyon, France. Material of Cycas rumphii Miq. was collected from plants cultivated in the New York Botanical Garden. Material of Ginkgo biloba L. was collected from plants cultivated in the Arnold Arboretum (Harvard University). Material of Podocarpus reichei J. Buchholz \& N.E. Gray was collected by Francisco Vergara-Silva from plants cultivated at Universidad Nacional Autónoma de México (Mexico City, Mexico). Taxus globosa Schltdl. was wild-collected by Francisco Vergara-Silva at El Chico (Hidalgo, Mexico). Material of Pseudotsuga menziesii (Mirb.) Franco was collected from plants cultivated at UC Davis.

\section{Data collection: molecular work}

Molecular cloning of YABBY cDNAs from fresh material of C. rumphii, G. biloba, G. gnemon, P. menziesii, P. reichei, and T. globosa was performed by PCR amplification. Total RNA was isolated from vegetative or reproductive tissues by the method of Chang et al. (Chang et al., 1993). First strand cDNA was synthesized using RevertAid $^{\mathrm{TM}}$ M-MuLV Reverse Transcriptase (Fermentas, Burlington, Canada). Partial fragments of YABBY cDNAs were amplified by using the degenerate primers 5'-TTGGATACAGTGACAGTGAAATGYGGNCAITG and 5'-TGCCCAATTTTTTGCTGCTGC. Alternatively, two nested PCR reactions were performed (using first strand cDNA as template) with one forward primer (5'GTIACIGTIMGITGYGGICAYTG) and two reverse primers (outer 5': 5'GCCCARTTYTTIGCIGC and inner 5': 5'- AAICKRAARTAIGCIGAIGGIAC) (Floyd et al., 2006). cDNA sequences were completed by 5' and 3' RACE using the MARATHON cDNA amplification kit (Clontech, Palo Alto, USA) or the SMART RACE kit (Clontech, Palo Alto, USA). For E. distachya and G. biloba, the cloning of 
YABBY sequences was performed by screening reproductive tissue cDNA libraries (Finet et al., 2013).

\section{Data collection: bioinformatic work}

YABBY genes were identified in available transcriptomes by tBLASTn using a set of selected A. thaliana YABBY genes as a probe. The accession numbers of all the sequences used in this study are indicated in Supplementary Table S1. Species of origin are indicated by the following prefixes Aas: Asparagus asparagoides, Aba: Abies balsamea, Ach: Annona cherimola, Afi: Aristolochia fimbriata, Afo: Aquilegia formosa, Ala: Abies lasiocarpa, Ama: Antirrhinum majus, Asq: Annona squamosa, Ath: Arabidopsis thaliana, Atr: Amborella trichopoda, Cat: Cedrus atlantica, Cca: Cabomba caroliniana, Cch: Cephalotaxus harringtonia, Cja: Cryptomeria japonica, Cmi: Cycas micholitzii, Cru: Cycas rumphii, Cse: Chloranthus serratus, Eca: Eschscholzia californica, Edi: Ephedra distachya, Gbi: Ginkgo biloba, Ggn: Gnetum gnemon, Ghi: Gossypium hirsutum, Gro: Grevillea robusta, Ltu: Liriodendron tulipifera, Mgr: Magnolia grandiflora, Nad: Nuphar advena, Nal: Nymphaea alba, Nala: Nepenthes alata, Nco: Nymphaea colorata, Nja: Nuphar japonica, Osa: Oryza sativa, Pam: Persea americana, Pgl: Picea glauca, Phy: Petunia hybrida, Pla: Pinus lambertiana, Pma: Podocarpus macrophyllus, Pme: Pseudotsuga menziesii, Ppi: Pinus pinaster, Pre: Podocarpus reichei, Psi: Picea sitchensis, Pta: Pinus taeda, She: Saruma henryi, Sly: Solanum lycopersicum, Sve: Sciadopitys verticillata, Tba: Taxus baccata, Tcu: Taxus cuspidata, Tgl: Taxus globosa, Vvi: Vitis vinifera, Wmi: Welwitschia mirabilis, Zva: Zamia vazquezii. The novel sequences generated for this analysis have been deposited in the EMBL database with the following accession numbers: LN871570 (CruYABB), LN871571 (EdiYABB), LN871572 (EdiYABD), LN871573 (GbiYAB1B), LN871574 (GbiYAB2B), LN871575 (GbiYABC), LN871576 (PmeYABA), LN871577 (PmeYABC), LN871578 (PreYABB), LN871579 (PreYABD), LN871580 (TglYAB1A), LN871581 (TglYAB1C), LN871582 (ZvaYABB).

\section{Phylogenetic analysis}

Nucleotide sequences were aligned with MUSCLE (Edgar, 2004), manually adjusted, and selected blocks were used for phylogenetic reconstruction. Maximum-likelihood (ML) searches were performed using RAxML v.8.1 (Stamatakis, 2014) under the sitehomogeneous GTR $+\Gamma$ model. 100 bootstrap replicates were conducted for support 
estimation. To detect potential impact of long-branch attraction, we also employed the site-heterogeneous CAT-GTR model implemented in PhyloBayes v.3.3 (Lartillot et al., 2009). We ran two independent chains for at least 10,000 cycles, discarded the first 1,000 cycles as burn-in, and recovered no major topological changes with regard to ML tree. We also evaluated the potential influence of compositional heterogeneity in the dataset by a posterior predictive test based on the PhyloBayes analysis. Taxa with high compositional heterogeneity (Z-score >2) were removed for the 83sequence analysis. For the 84-sequence analysis, coding nucleotide sequences were manually aligned as amino acid translations using Se-Al v2.0 (Rambaut, 2002). We excluded ambiguously aligned sequence to produce an alignment of 113 amino acid characters in 84 YABBY gene sequences. The corresponding 339 nucleotides were used for subsequent Bayesian analysis. Bayesian phylogenetic analysis was performed using Mr. Bayes 3.2.1, run on multiple parallel processors (Ronquist et al., 2012). The characters were partitioned by position in codon allowing for estimation of different substitution rates at each position within the codons with a general time reversible model applied. The Bayesian analyses were run for 10,000,000 generations, which was sufficient for convergence of the two simultaneous runs - convergence took 653,000 generations. For the burn-in phase $25 \%$ of the total number of saved trees was discarded. The different nucleotide sequence alignments and tree files are downloadable from TreeBASE (http://purl.org/phylo/treebase/phylows/study/TB2:S18258).

\section{Histology}

Ephedra tissues were fixed in FAA (formaldehyde - acetic acid - ethanol) or 1\% paraformaldehyde (PFA) overnight and then dehydrated through an ethanol series to $100 \%$ ethanol. The ethanol was gradually replaced with Histo-Clear II (National Diagnostics). The Histo-clear was gradually replaced with Paraplast X-tra (Fisher Scientific) at $60^{\circ} \mathrm{C}$. Embedded specimens were sectioned at 8-10 $\mu \mathrm{m}$ and dried overnight at $37^{\circ} \mathrm{C}$. Tissues were hydrated through an ethanol series to $\mathrm{H}_{2} \mathrm{O}$ and the slides were stained in $0.1 \%$ toluidine blue. Images were captured by using a Leica MZ12 binocular equipped with a Leica DFC320 digital camera. Shoot apices (including young leaves) of Ginkgo and Pseudotsuga were fixed in a solution of $1.5 \%$ glutaraldehyde, $1 \%$ paraformaldehyde, and 4\% acrolein in Pipes buffer (84 mM 
Pipes, $8.4 \mathrm{mM}$ EGTA and $\left.1.6 \mathrm{mM} \mathrm{MgSO}_{4}\right)$ at $\mathrm{pH}$ 6.8. Specimens were left in fixative a minimum of 24 hours, then rinsed in Pipes buffer and dehydrated through an ethanol series to $95 \%$ ethanol. Specimens were then infiltrated with catalyzed monomer A of the JB-4 embedding kit (Polysciences), and embedded in an oxygen-free environment following the basic protocol provided with the kit. Blocks were serially sectioned at 5 $\mu \mathrm{m}$ on an HM 355S (Microm) rotary microtome using glass knives. Slides were stained in $0.1 \%$ toluidine blue, examined, and photographed on a Zeiss Axioskop microscope equipped with a Zeiss Axiocam digital camera using bright field microscopy.

\section{In situ hybridization}

Tissues were fixed in FAA or PFA and subsequently embedded and sectioned as detailed above. Digoxigenin-labeled antisense and sense RNA probes were prepared from full-length or partial cDNA clones. Our prehybridization, hybridization, and posthybridization procedures were based on those of Jackson et al. with some modifications (Jackson, 1991). Whole mount in situ hybridizations were carried out as described by Hejátko et al. (Hejátko et al., 2006). Slides were examined and photographed on a Leica MZ12 binocular equipped with a Leica DFC320 digital camera (Ephedra), a Zeiss Axioskop microscope equipped with a Zeiss Axiocam digital camera (Ginkgo and Pseudotsuga), or a Zeiss Lumar stereomicroscope equipped with a Zeiss Axiocam digital camera (whole-mount Ginkgo).

\section{Results and Discussion}

\section{The ancestral YABBY complement}

Resolution of YABBY family evolution is essential to understanding the contribution that YABBY genes have made to seed plant evolution. However, the paucity of phylogenetically informative characters in YABBY genes and the high degree of similarity between family members have made it difficult to reconstruct robust phylogenies of the YABBY family across large evolutionary distances. In order to address this issue and to better understand YABBY diversification across all seed plants, we compiled a dataset of 43 YABBY sequences from across gymnosperms. 
Our phylogenetic analyses indicate that YABBY homologues in gymnosperms are resolved into four distinct clades. The clades include sequences from most of the major lineages of gymnosperm taxa (Figure 1A). Whereas clades A, C, and D are well supported, the branch leading to the clade B has only weak support. However, that clade B consists of sequences from each of the major gymnosperm clades and reflects their accepted phylogenetic relationships (Lu et al., 2014) provides additional support for the clade. The grouping of Coniferales I, Coniferales II, and Gnetales within clade $\mathrm{B}$ is additionally supported by a 15-bp deletion in the region encoding the YABBY domain (Figure 1B). High variability in the relative position of Cycadales and Ginkgoales at the base of clade B could explain the poor statistical values observed. These four clades indicate that at least four YABBY genes were present in the last common ancestor of extant gymnosperms.

We next investigated the relative relationships of angiosperm and gymnosperm YABBY clades. We assembled a dataset of 107 YABBY sequences, including the list of 43 gymnosperm sequences (Figure 1A) compiled in the present work and sequences spanning the phylogenetic diversity of angiosperms. Phylogenetic analyses of the 107-sequence dataset identify gymnosperm and angiosperm YABBY genes as two distinct monophyletic groups (Figure 2A and S1A). Because composition biases among synonymous substitutions have been shown to potentially generate conflicting phylogenies (Cox et al., 2014), we identified and removed sequences having such a bias. Phylogenetic analysis of the resulting 83-sequence dataset supports monophyly of gymnosperm YABBY genes (Figure S1B). We also observed a few sequences with faster rates of molecular evolution. However, phylogenetic analysis of a dataset in which sequences with longer branches have been removed supports an alternative topology, in which gymnosperm YABBY genes were not resolved as monophyletic. In this analysis, a gymnosperm clade comprised of clades B and D is sister to an angiosperm clade composed of CRC and FIL/YAB3, whereas the two gymnosperm clades $\mathrm{A}$ and $\mathrm{C}$ form a polytomy with the angiosperm clade composed of the YAB5, YAB2, and INO clades (Figures 2B and S1C).

Given there is not an appropriate outgroup sequence to root the trees, we rooted the trees based on parsimony, minimizing the numbers of gene gains and losses required assuming both angiosperm and gymnosperm taxa are monophyletic groups. Based on 
this rooting, the two alternative topologies lead to different evolutionary scenarios. Considering gymnosperm YABBYs as paraphyletic, with gymnosperm sequences that group with two distinct angiosperm clades (Figure 2B), would suggest the presence of two YABBY genes in the last common ancestor of extant seed plants. Conversely, the monophyly of gymnosperm YABBY genes (Figure 2A) would suggest that all spermatophyte YABBY genes derived from a single gene in the common ancestor of extant seed plants and that diversification of the gene family proceeded independently in both angiosperms and gymnosperms. Previous analyses with very limited gymnosperm datasets also suggested that gymnosperm YABBY genes could be either monophyletic (Bartholmes et al., 2012) or paraphyletic (Yamada et al., 2011). It has been proposed that prior to the divergence of angiosperms and gymnosperms, a whole genome duplication occurred in the ancestral seed plant and another in the ancestral angiosperm (Jiao et al., 2011). In addition, analyses of individual gene families that have included homologs from all major lineages of land plants suggest that multiple paralogs arose in the seed-plant common ancestor after the divergence of monilophytes: ARFs (Finet et al., 2013), class III HD-Zips (Floyd et al., 2006)(Prigge and Clark, 2006), class IV HD-Zips (Zalewski et al., 2013), LBDs (Chanderbali et al., 2015), PINs (Bennett et al., 2014). There is less evidence for any recent whole genome duplications within the gymnosperm lineage, although analyses to date have focused only on the conifers (Pavy et al., 2012)(Nystedt et al., 2013). Either of the two alternative topologies proposed could be consistent with this history of genome duplication, although if gymnosperm and angiosperm YABBY genes are both monophyletic, it would imply a gene loss in the seed-plant common ancestor and following the hypothesized genome duplication in the seed plant common ancestor.

The relationship among the angiosperm YABBY genes is very robust in our analyses with regard to any common phylogenetic bias we tested for. We can distinguish a first cluster composed of the clades CRC and FIL, and a second cluster in which the clades YAB5, YAB2, and INO group together. Noticeably, $C R C$ and $F I L$ share the common feature of being 'strong' patterning genes by promoting abaxial cell fate (Eshed et al., 1999)(Sawa et al., 1999)(Siegfried et al., 1999), whereas YAB2, YAB5, and INO seem to have a milder effect on patterning but a stronger role in tissue growth (Siegfried et al., 1999)(Eshed et al., 2004)(Golz et al., 2004)(Sarojam et al., 2010). Furthermore, 
the two genes directing the development of angiosperm specific innovations, the carpel $(C R C)$ and ovule outer integument $(I N O)$, were derived from different YABBY gene ancestors. However, this evolutionary scenario of the YABBY family within angiosperms is not congruent with conclusions from other phylogenetic studies (Yamada et al., 2011)(Bartholmes et al., 2012), where a clade comprised of FIL, CRC, and INO was observed. Given the inherent instability in tree topology depending upon inclusion/exclusion of taxa and mode of analysis, the phylogenetic relationships between the gymnosperm and angiosperm YABBY genes presented here remain hypotheses for future investigation.

There are reports of YABBY genes in other lineages of eukaryotes, such as within the Chlorophyta (Worden et al., 2009). We searched GenBank for sequences similar to YABBY genes in species other than land plants and found similar sequences in both the Chlorophyta and fungi. However, the region of similarity was restricted to the helix-loop-helix portion of the YABBY domain, and in none of the sequences was a $\mathrm{C} 2 \mathrm{C} 2$ zinc finger domain found, either amino or carboxyl to the region of similarity within the helix-loop-helix domain. Furthermore, the putative helix-loop-helix domains were not conserved across taxa within the Chlorophyta, in which there are 31 pairwise amino acid differences between the sequences in Ostreococcus and Micromonas (Figure S2A). In contrast, other than $C R C$ and $I N O$, which have evolved specialized functions in the carpel and ovule of angiosperms, all the other seed plant YABBY genes have accumulated fewer than 10 mutations (and most five or less) from the predicted ancestral seed plant YABBY amino acid sequence (Figure S2A). Phylogenetic analyses place the fungal and Chlorophyta sequences approximately equidistant between seed plant YABBY sequences and canonical HMG box sequences (Figure S2B). While it remains possible that the fungal and Chlorophyta sequences may be more directly evolutionarily related to seed plant YABBY genes, it seems equally, or perhaps more likely, that they represent independent derivations from ancestral HMG box sequences.

\section{YABBY genes and the evolution of seed-plant leaves}

While most genes that direct leaf development in angiosperms predate the evolutionary origin of seed-plant leaves, the YABBY genes originated along the 
lineage leading to seed plants (Floyd and Bowman, 2007)(Sarojam et al., 2010). In Arabidopsis, simultaneous loss-of-function of the four vegetative YABBY genes (FIL, YAB2, YAB3, and YAB5) results in polarity defects, loss of lamina expansion, and reactivation of shoot meristem genetic programs (Sarojam et al., 2010). Those findings led some authors to propose that YABBY genes act to transform an ancestral shoot-specific network into a leaf-specific one (Sarojam et al., 2010)(Floyd and Bowman, 2010).

We performed in situ hybridization experiments to investigate expression patterns of YABBY genes in vegetative tissues in Ginkgo and Pseudotsuga. In Pseudotsuga, expression of PmeYABC does not show any clear evidence of polar patterning. The staining is visible throughout the provascular strand in both leaf primordia and young leaves (Figures 3D and 3E). In contrast to PmeYABC expression, in Ginkgo, strong expression of $G b i Y A B C$ is evident on the abaxial side of leaf primordia (Figures 4B and $4 C$ ). Staining from the GbiYABC probe is also visible in the leaf primordium provasculature (Figure 4B). The expression pattern of Ginkgo gene GbiYAB1B is very similar to GbiYABC (Figures $4 \mathrm{~F}$ and $4 \mathrm{G}$ ), being abaxial in young primordia and maintaining a similar, though slightly weaker pattern in older primordia. Expression of $G b i Y A B 2 B$ is also restricted to the abaxial side of the youngest leaf primordia (Figures 4D and 4E). In older primordia, its expression remains abaxial but is restricted to distal, marginal positions (Figure 4E). In older leaf primordial, staining of GbiYAB1B and GbiYAB2B appears to be complementary, with the former present in the basal-abaxial domain and the later present in the distal-abaxial domain. Phylogenetic analysis identified $G b i Y A B 1 B$ and $G b i Y A B 2 B$ as paralogues that originated from a gene duplication event unique to the Ginkgo lineage (Figure 1A). In situ expression data are consistent with subfunctionalization following that duplication event.

The three Ginkgo genes residing in gymnosperm clades B and C appear to be expressed abaxially in leaves, similar to expression of YABBY genes in the angiosperm YAB2, YAB5, and FIL/YAB3 clades. Based on parsimony, this phylogenetic distribution suggests that this pattern may represent the ancestral expression pattern of the entire YABBY family, irrespective of the two alternative evolutionary scenarios presented, implying that other expression patterns observed are 
derived. Interestingly, expression pattern of $G b i Y A B C$, which stains the outside (abaxial domain) of the bifurcating lamina and petiole (Figure 4B), appears to be complementary to the expression domain of the Ginkgo class III HD-zip gene, $G b C 3 H D Z 1$, which stains in the inside (adaxial domain) of the notch of the bifurcation (Floyd and Bowman, 2006). This observation suggests that the mutual antagonism between class III HD-ZIP and YABBY genes characterized in angiosperms could have arisen prior to the split between gymnosperms and angiosperms.

There are some interesting differences in YABBY gene expression between Pseudotsuga, Ginkgo, and Arabidopsis. In contrast to Arabidopsis and Gingko, YABBY expression in the conifer Pseudotsuga (PmeYABC) was not clearly polar in young leaf primordia. Pseudostuga leaves, like other Pinaceae, are linear and needlelike. The needle-like leaves of Pseudotsuga differ from leaves of Ginkgo and flowering plants in that they lack laminar expansion and clear dorsoventral organization of mesophyll and epidermal tissues (Figure 3C). Based upon the parsimony criterion, the needle-like leaves of conifers were likely derived from ancestors with more broadly laminate leaves. The evolution of conifer leaves was likely the result of numerous changes to the leaf developmental program. Our data suggest that at least one such change may have been a loss of YABBY expression polarity during leaf development.

In Arabidopsis, FIL/YAB3 expression in leaf primordia becomes restricted to marginal positions reflecting the early differentiation of the distal end of the developing leaf (Siegfried et al., 1999). In contrast, Ginkgo leaves continue to grow throughout their development by distal meristematic activity (Bower, 1884)(Mundry and Stuitzel, 2004). Expression of GBiYAB2B is limited to abaxial-distal regions in older leaf primordia. This expression pattern is unlike that of FIL/YAB3 and suggests that YABBY expression is associated with laminar expansion in gymnosperm leaves with distal (apical) and angiosperm leaves with marginal meristematic activity.

\section{YABBY genes and the evolution of seed plant ovules}

We performed in situ hybridization experiments to investigate expression patterns of YABBY genes in female reproductive structures in Ephedra. EdiYABD transcripts 
were detected in ovule integuments and at the base of sterile bracts (Figure 5B). The staining is throughout the innermost bract but is restricted to the abaxial side in outer bracts (Figure 5C). The other gene investigated, EdiYABB, is expressed throughout the ovule integument, with a clear enrichment in the outermost cell layer of the integument (Figures 5D and 5E). EdiYABB is also weakly expressed at the base of sterile bracts and may be more strongly expressed in the abaxial side.

Gymnosperm ovules have a single integument with the micropyle positioned opposite the stalk (orthotropous). Most angiosperms, including all ANA-grade taxa, have two integuments, and the ovule is commonly bent over (anatropous), positioning the micropyle close to the stalk. The second integument is not simply a reiteration of the first; the two clearly have different attributes and evolutionary origins. The inner integument most likely originated directly from the fusion of telomes (Zimmermann, 1952) or sporangiophores (Benson, 1904)(Kenrick and Crane, 1997). However, the origin of the second integument remains one of the cryptic and controversial questions concerning the origin of the flowering plants. It has been proposed that the outer integument evolved later, from a cupule, a leaf-like structure surrounding one or more ovules (Stebbins, 1974)(Gasser et al., 1998). Consistent with an origin from a leaf, in angiosperms, the YABBY gene INO is a molecular marker specific to the outer integument of ovules (Villanueva et al., 1999) and development of the inner integument does not require YABBY expression or function. In the present study, we show that the two genes $E \operatorname{diY} A B B$ and $E \operatorname{diYABD}$ are strongly expressed in the ovule integument in Ephedra. Our finding of YABBY expression in the single integument of a gymnosperm is surprising in the light of the classical view that the gymnosperm integument is homologous to the inner integument in angiosperms. However, Gnetales female cones are known to show highly derived characters. Investigation of gene expression in ovules of other non-flowering plants is needed to determine whether YABBY genes are universally associated with the gymnosperm integument. These data should be informative with respect to the evolution of ovule integuments during seed plant evolution.

In summary, either one or two YABBY genes were present in the last common ancestor of extant seed plants. That some YABBY genes are expressed in a polar (abaxial) manner in leaves and female cones in gymnosperms suggests that in the last common ancestor of seed plants at least one member of the YABBY gene 
family was likely expressed in a polar manner. We propose that YABBY genes already acted as polarity genes in the last common ancestor of extant seed plants, and as polarity is intimately linked to lamina expansion, that YABBY gene function is associated with the evolution of laminar leaves in seed plants.

\section{Acknowledgments}

We thank Jean-Pierre Grienay (Lyon Botanic Garden), Andrew Groover and Dave Johnson (USDA Institute of Forest Genetics, Placerville) for assistance with obtaining plants used in this study. We thank Eric Brenner (formerly of New York Botanical Garden) for providing Cycas rumphii cDNA libraries and Francisco Vergara-Silva for providing RNA samples of Taxus globosa and Podocarpus reichei. We thank Judith Lucas and Karen Yip for assistance with cloning YABBY cDNAs from Pseudotsuga menziesii and Ginkgo biloba. We thank LinHua Sun for assistance with phylogenetic analyses. S.K.F. was supported in part by the UC Davis Katherine Esau postdoctoral fellowship and the Australian Research Council Future Fellowship FT100100763. Work was also supported by the Priority Academic Program Development of Jiangsu Higher Education Institutions and Natural Science Foundation of China for Talents Training in Basic Science (Project J1103507) to B.Z., and Australian Research Council Discovery Project DP110100070 to J.L.B.

\section{References}

Banks, J.A., Nishiyama, T., Hasebe, M., Bowman, J.L., Gribskov, M., dePamphilis, C., Albert, V.A., Aono, N., Aoyama, T., Ambrose, B.A., et al. 2011. The Selaginella genome identifies genetic changes associated with the evolution of vascular plants. Science 332, 960-963.

Bartholmes, C., Hidalgo, O., and Gleissberg, S. 2012. Evolution of the YABBY gene family with emphasis on the basal eudicot Eschscholzia californica (Papaveraceae). Plant Biol. 14, 11-23.

Baum, S.F., Eshed, Y., and Bowman, J.L. 2001. The Arabidopsis nectary is an ABCindependent floral structure. Development 128, 4657-4667.

Bennett, T., Brockington, S.F., Rothfels, C., Graham, S.W., Stevenson, D., Kutchan, T., Rolf, M., Thomas, P., Wong, G.K.S., Leyser, O., et al. 2014. Paralogous radiations of PIN proteins with multiple origins of noncanonical PIN structure. Mol. Biol. Evol. 31, 2042-2060. 
Benson, M. 1904. Telangium scotti, a new species of Telangium (Calymmatotheca) showing structure. Ann. Bot. 78, 161-177.

Bower, F. 1884. On the comparative morphology of the leaf in the vascular cryptograms and gymnosperms. Philos. Trans. R. Soc. B 175, 565-615.

Bowman, J.L. 2000. The YABBY gene family and abaxial cell fate. Curr. Opin. Plant Biol. 3, 17-22.

Bowman, J.L., and Smyth, D.R. 1999. CRABS CLAW, a gene that regulates carpel and nectary development in Arabidopsis, encodes a novel protein with zinc finger and helix-loop-helix domains. Development 126, 2387-2396.

Chanderbali, A.S., He, F., Soltis, P.S., and Soltis, D.E. 2015. Out of the Water: Origin and Diversification of the LBD Gene Family. Mol. Biol. Evol.

Chang, S., Puryear, J., and Cairney, J. 1993. A simple and efficient method for isolating RNA from pine trees. Plant Mol. Biol. Report. 11, 113-116.

Cox, C.J., Li, B., Foster, P.G., Embley, T.M., and Civan, P. 2014. Conflicting Phylogenies for Early Land Plants are Caused by Composition Biases among Synonymous Substitutions. Syst. Biol. 63, 272-279.

Edgar, R.C. 2004. MUSCLE: multiple sequence alignment with high accuracy and high throughput. Nucl. Acids Res. 32, 1792-1797.

Eshed, Y., Baum, S.F., and Bowman, J.L. 1999. Distinct mechanisms promote polarity establishment in carpels of Arabidopsis. Cell 99, 199-209.

Eshed, Y., Izhaki, A., Baum, S.F., Floyd, S.K., and Bowman, J.L. 2004. Asymmetric leaf development and blade expansion in Arabidopsis are mediated by KANADI and YABBY activities. Development 131, 2997-3006.

Finet, C., Berne-Dedieu, A., Scutt, C.P., and Marlétaz, F. 2013. Evolution of the ARF gene family in land plants: Old domains, new tricks. Mol. Biol. Evol. 30, 45-56.

Floyd, S.K., and Bowman, J.L. 2006. Distinct Developmental Mechanisms Reflect the Independent Origins of Leaves in Vascular Plants. Curr. Biol. 16, 1911-1917.

Floyd, S.K., and Bowman, J.L. 2007. The Ancestral Developmental Tool Kit of Land Plants. Int. J. Plant Sci. 168, 1-35.

Floyd, S.K., and Bowman, J.L. 2010. Gene expression patterns in seed plant shoot meristems and leaves: homoplasy or homology? J. Plant Res. 123, 43-55.

Floyd, S.K., Zalewski, C.S., and Bowman, J.L. 2006. Evolution of class III homeodomain-leucine zipper genes in streptophytes. Genetics 173, 373-388.

Fourquin, C., Vinauger-Douard, M., Fogliani, B., Dumas, C., and Scutt, C.P. 2005. Evidence that CRABS CLAW and TOUSLED have conserved their roles in carpel 
development since the ancestor of the extant angiosperms. Proc. Natl. Acad. Sci. U. S. A. $102,4649-4654$.

Gasser, C.S., Broadhvest, J., and Hauser, B.A. 1998. GENETIC ANALYSIS OF OVULE DEVELOPMENT. Annu. Rev. Plant Physiol. Plant Mol. Biol. 49, 1-24.

Golz, J.F., Roccaro, M., Kuzoff, R., and Hudson, A. 2004. GRAMINIFOLIA promotes growth and polarity of Antirrhinum leaves. Development 131, 3661-3670.

Hejátko, J., Blilou, I., Brewer, P.B., Friml, J., Scheres, B., and Benková, E. 2006. In situ hybridization technique for mRNA detection in whole mount Arabidopsis samples. Nat. Protoc. 1, 1939-1946.

Jackson, D. 1991. in situ hybridisation in plants. In Molecular Plant Pathology, A Practical Approach, D. Bowles, S. Gurr, and M. McPherson, eds. (Oxford University Press, UK),

Jiao, Y., Wickett, N.J., Ayyampalayam, S., Chanderbali, A.S., Landherr, L., Ralph, P.E., Tomsho, L.P., Hu, Y., Liang, H., Soltis, P.S., et al. 2011. Ancestral polyploidy in seed plants and angiosperms. Nature 473, 97-100.

Juarez, M.T., Kui, J.S., Thomas, J., Heller, B.A., and Timmermans, M.C.P. 2004a. microRNA-mediated repression of rolled leaf1 specifies maize leaf polarity. Nature $428,84-88$.

Juarez, M.T., Twigg, R.W., and Timmermans, M.C.P. 2004b. Specification of adaxial cell fate during maize leaf development. Development 131, 4533-4544.

Kenrick, P., and Crane, P. 1997. The origin and early diversification of land plants: a cladistic study. (Washington, DC).

Lartillot, N., Lepage, T., and Blanquart, S. 2009. PhyloBayes 3: A Bayesian software package for phylogenetic reconstruction and molecular dating. Bioinformatics 25, 2286-2288.

Lee, J.-Y., Baum, S.F., Oh, S.-H., Jiang, C.-Z., Chen, J.-C., and Bowman, J.L. 2005. Recruitment of CRABS CLAW to promote nectary development within the eudicot clade. Development 132, 5021-5032.

Lora, J., Hormaza, J.I., Herrero, M., and Gasser, C.S. 2011. Seedless fruits and the disruption of a conserved genetic pathway in angiosperm ovule development. Proc. Natl. Acad. Sci. U. S. A. 108, 5461-5465.

Lu, Y., Ran, J.-H., Guo, D.-M., Yang, Z.-Y., and Wang, X.-Q. 2014. Phylogeny and Divergence Times of Gymnosperms Inferred from Single-Copy Nuclear Genes. PLoS One 9 ,.

McAbee, J.M., Kuzoff, R.K., and Gasser, C.S. 2005. Mechanisms of derived unitegmy among Impatiens species. Plant Cell 17, 1674-1684. 
Mundry, M., and Stützel, T. 2004. Morphogenesis of leaves and cones of male shortshoots of Ginkgo biloba L. Flora - Morphol. Distrib. Funct. Ecol. Plants 199, 437452.

Nystedt, B., Street, N.R., Wetterbom, A., Zuccolo, A., Lin, Y.-C., Scofield, D.G., Vezzi, F., Delhomme, N., Giacomello, S., Alexeyenko, A., et al. 2013. The Norway spruce genome sequence and conifer genome evolution. Nature 497, 579-584.

Orashakova, S., Lange, M., Lange, S., Wege, S., and Becker, A. 2009. The CRABS CLAW ortholog from California poppy (Eschscholzia californica, Papaveraceae), EcCRC, is involved in floral meristem termination, gynoecium differentiation and ovule initiation. Plant J. 58, 682-693.

Pavy, N., Pelgas, B., Laroche, J., Rigault, P., Isabel, N., and Bousquet, J. 2012. A spruce gene map infers ancient plant genome reshuffling and subsequent slow evolution in the gymnosperm lineage leading to extant conifers. BMC Biol. 10, 84.

Prigge, M.J., and Clark, S.E. 2006. Evolution of the class III HD-Zip gene family in land plants. Evol. Dev. 8, 350-361.

Rambaut, A. 2002. Se-Al v. 2.0a11: sequence alignment program. (http://tree.bio.ed.ac.uk/software/seal/).

Rensing, S.A., Lang, D., Zimmer, A.D., Terry, A., Salamov, A., Shapiro, H., Nishiyama, T., Perroud, P.-F., Lindquist, E.A., Kamisugi, Y., et al. 2008. The Physcomitrella genome reveals evolutionary insights into the conquest of land by plants. Science 319, 64-69.

Ronquist, F., Teslenko, M., Van Der Mark, P., Ayres, D.L., Darling, A., Höhna, S., Larget, B., Liu, L., Suchard, M.A., and Huelsenbeck, J.P. 2012. Mrbayes 3.2: Efficient bayesian phylogenetic inference and model choice across a large model space. Syst. Biol. 61, 539-542.

Sarojam, R., Sappl, P.G., Goldshmidt, A., Efroni, I., Floyd, S.K., Eshed, Y., and Bowman, J.L. 2010. Differentiating Arabidopsis shoots from leaves by combined YABBY activities. Plant Cell 22, 2113-2130.

Sawa, S., Ito, T., Shimura, Y., and Okada, K. 1999. FILAMENTOUS FLOWER controls the formation and development of arabidopsis inflorescences and floral meristems. Plant Cell 11, 69-86.

Siegfried, K.R., Eshed, Y., Baum, S.F., Otsuga, D., Drews, G.N., and Bowman, J.L. 1999. Members of the YABBY gene family specify abaxial cell fate in Arabidopsis. Development 126, 4117-4128.

Stamatakis, A. 2014. RAxML version 8: A tool for phylogenetic analysis and postanalysis of large phylogenies. Bioinformatics 30, 1312-1313.

Stebbins, G.L. 1974. Flowering plants: evolution above the species level. (Cambridge, Massachusetts, Harvard University Press). 
Villanueva, J.M., Broadhvest, J., Hauser, B.A., Meister, R.J., Schneitz, K., and Gasser, C.S. 1999. INNER NO OUTER regulates abaxial-adaxial patterning in Arabidopsis ovules. Genes Dev. 13, 3160-3169.

Worden, A.Z., Lee, J.-H., Mock, T., Rouzé, P., Simmons, M.P., Aerts, A.L., Allen, A.E., Cuvelier, M.L., Derelle, E., Everett, M. V, et al. 2009. Green evolution and dynamic adaptations revealed by genomes of the marine picoeukaryotes Micromonas. Science 324, 268-272.

Yamada, T., Ito, M., and Kato, M. 2003. Expression pattern of INNER NO OUTER homologue in Nymphaea (water lily family, Nymphaeaceae). Dev. Genes Evol. 213, $510-513$.

Yamada, T., Ito, M., and Kato, M. 2004. YABBY2-homologue expression in lateral organs of Amborella trichopoda (Amborellaceae). Int. J. Plant Sci. 165, 917-924.

Yamada, T., Yokota, S., Hirayama, Y., Imaichi, R., Kato, M., and Gasser, C.S. 2011. Ancestral expression patterns and evolutionary diversification of YABBY genes in angiosperms. Plant J. 67, 26-36.

Yamaguchi, T., Nagasawa, N., Kawasaki, S., Matsuoka, M., Nagato, Y., and Hirano, H.-Y. 2004. The YABBY gene DROOPING LEAF regulates carpel specification and midrib development in Oryza sativa. Plant Cell 16, 500-509.

Zalewski, C.S., Floyd, S.K., Furumizu, C., Sakakibara, K., Stevenson, D.W., and Bowman, J.L. 2013. Evolution of the class IV HD-Zip gene family in streptophytes. Mol. Biol. Evol. 30, 2347-2365.

Zimmermann, W. 1952. Main results of the telome theory. Paleobot. 1, 456-470.

\section{Legends}

Figure 1. Phylogeny of YABBY genes in gymnosperms. (A) Phylogram of the 43sequence analyses. RAxML maximum-likelihood analyses and PhyloBayes Bayesian analyses were conducted under the GTR $+\Gamma$ and the CAT-GTR model, respectively. Support values obtained after 100 bootstrap replicates (BP) and Bayesian posterior probabilities (PP) are shown for all branches. Scale bar indicates number of changes per site.

Figure 2. Phylogeny of YABBY genes in seed plants. Phylogenetic analyses generate two alternative topologies. (A) Gymnosperm YABBY genes are paraphyletic, suggesting at least two YABBY genes in the common ancestor of extant 
seed plants. (B) Gymnosperm YABBY genes form a monophyletic clade, suggesting a single YABBY gene in the ancestor of extant seed plants.

Figure 3. In situ Hybridization of YABBY Gene in Pseudotsuga. Purple stain indicates the localized hybridization of the antisense RNA probe. Brown staining indicates the presence of oxidized tannins. (A) Longitudinal section through the $P$. menziesii shoot apex. (B) Transverse section through apex at the level of leaf initiation. Leaf primordia are labeled P0 (youngest) through P4 (oldest). (C) Cross section of leaf. (D, E) PmeYABC is expressed in the provascular strand in both leaf primordial and young leaves. Abbreviations: endo (endodermis), lf (leaf), lp (leaf primordium), pv (provascular tissue), res (resin duct), sam (shoot apical meristem), and $\mathrm{vb}$ (vascular bundle).

Figure 4. In situ Hybridization of YABBY Genes in Ginkgo. Purple stain indicates the localized hybridization of the antisense RNA probe. Brown staining indicates the presence of oxidized tannins. (A) Longitudinal section through the G. biloba shoot apex and leaf. (B) Leaf primordia showing $G b i Y A B C$ expression restricted to abaxial side. (C) In situ hybridization of whole-mount apices confirms the abaxial expression of GbiYABC in leaf primordia labeled P0-P3 from youngest to oldest. (D) GbiYAB2 expression is restricted to abaxial side of leaf primordia. (E) Expression of GbiYAB2 is still abaxially restricted in older leaf primordia, yet weaker than in youngest primordia. (F) Expression patterns of GbiYAB3 are very similar to those of GbiYAB2 at the shoot apex. Abbreviations: ab (abaxial), ad (adaxial), lf (leaf), lp (leaf primordium), pv (provascular tissue), and sam (shoot apical meristem).

Figure 5. In situ Hybridization of YABBY Genes in Ephedra. Purple stain indicates the localized hybridization of the antisense RNA probe. Brown staining indicates the presence of oxidized tannins. (A) Longitudinal section through the $E$. distachya female cone. The female cone usually bears whorls of bracts, among which only the uppermost whorl is fertile. Each ovule has a leathery outer envelope (chlamys) and a membranous inner envelope (integument). (B) Biovulate female cone showing EdiYAB1 expression in integument. (C) Sterile bracts showing EdiYAB1 expression in successive whorls of bracts. Staining throughout the innermost bract becomes restricted to the abaxial side in outer bracts. (D) Female cone showing 
EdiYAB2 expression in integument. (E) At higher magnification, EdiYAB2 shows a stronger expression in the outermost cell layer of integument. Abbreviations: $a b$ (abaxial), ad (adaxial), br (bract), ch (chlamys), int (integument), and nu (nucellus).

Table S1. List of YABBY sequences selected for phylogenetic analyses. *: Sequence Read Archive (SRA) experiment set reference used by NCBI, **: The 1000 Plants (1KP) Project.

Figure S1. (A) Phylogram of the 107-sequence analysis. RAxML maximumlikelihood analysis was conducted under the $G T R+\Gamma$ model. Support values obtained after 100 bootstrap replicates are shown for all branches. (B) Phylogram of the 83sequence analysis. PhyloBayes analysis was conducted under the CAT-GTR model. Numbers at branches indicate posterior probability values. (C) Bayesian phylogram of seed plant YABBY genes based on an alignment of 84 sequences. Numbers at branches indicate posterior probability values. Gymnosperm genes are paraphyletic if the tree is rooted, as shown, to maximize parsimony. Gymnosperm clades B and D are sister to an angiosperm clade comprised of FIL and CRC orthologs, while gymnosperm clades $\mathrm{A}$ and $\mathrm{C}$ form a polytomy with an angiosperm clade composed of INO, YAB2 and $Y A B 5$ orthologues.

Figure S2. YABBY-like sequences from taxa other than seed plants. (A) Sequence alignment of seed plant YABBY genes (one from each angiosperm and gymnosperm clade), fungal and chlorophyte YABBY-like sequences, and canonical HMG boxes. The ancestral seed plant YABBY box sequence is predicted from the sequences of extant seed plants. The number (n) of amino acid substitutions predicted for each of the genes from the predicted ancestral seed plant sequence is listed at the far right. There are 31 amino acid differences (out of 58 positions) between the two Chlorophyta genes. (B) Bayesian phylogram constructed using amino acid alignment in $\mathrm{A}$. 


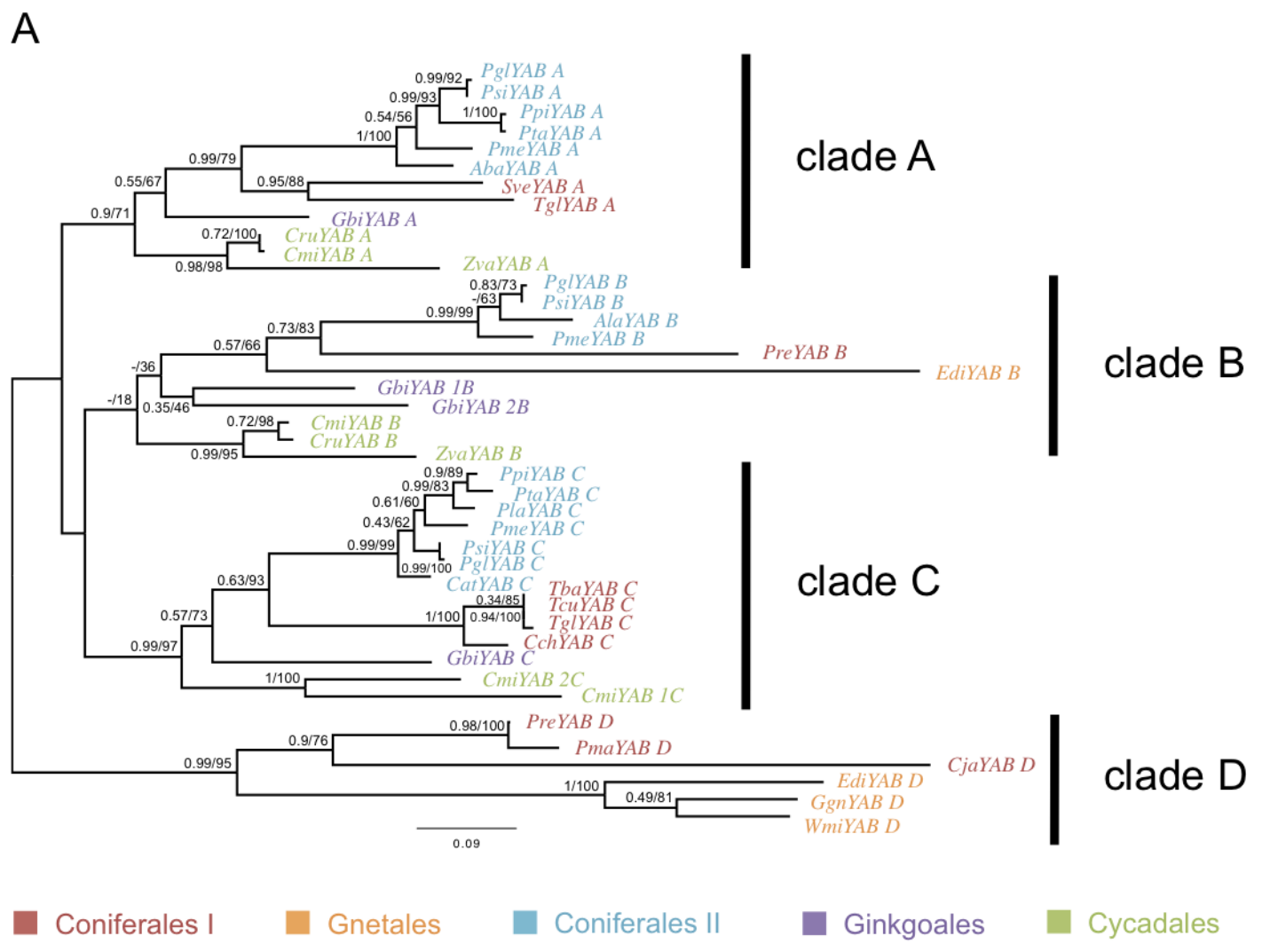

B

YABBY domain

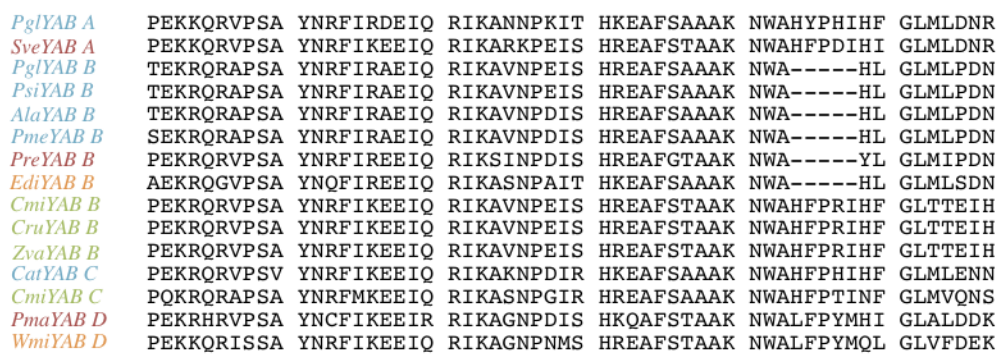

Figure 1 

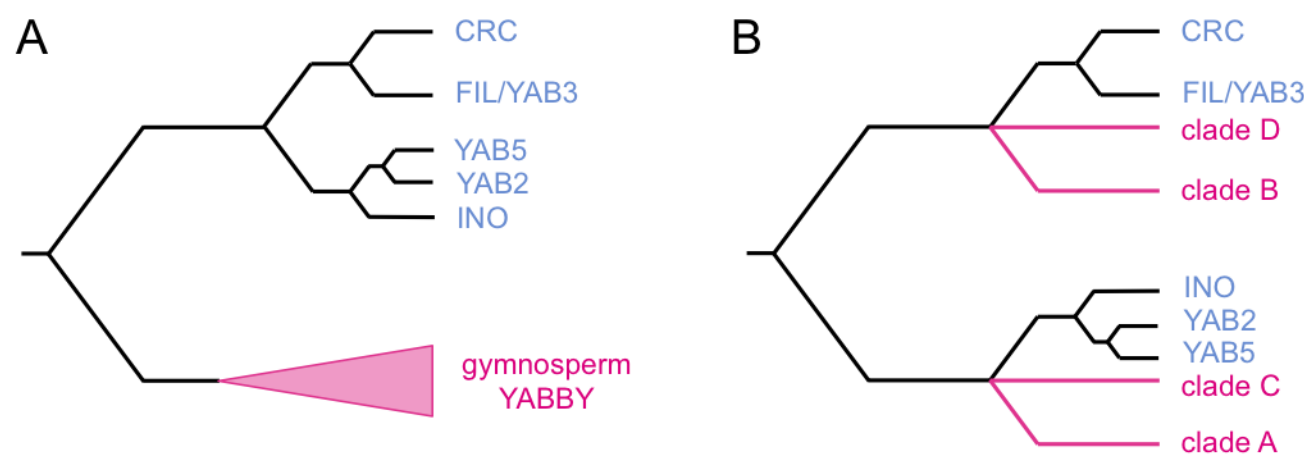

Figure 2

This article is protected by copyright. All rights reserved 

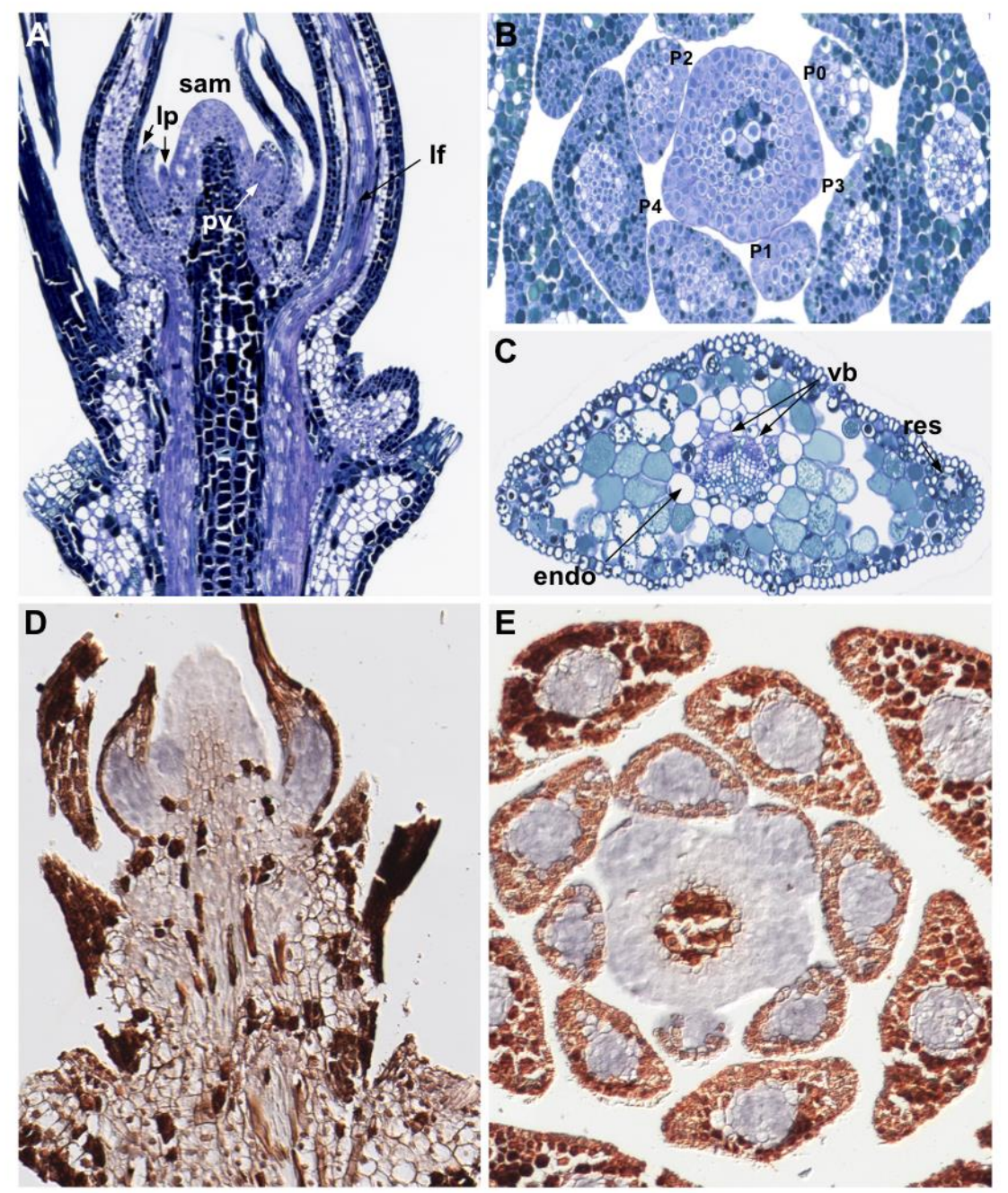

Figure 3 


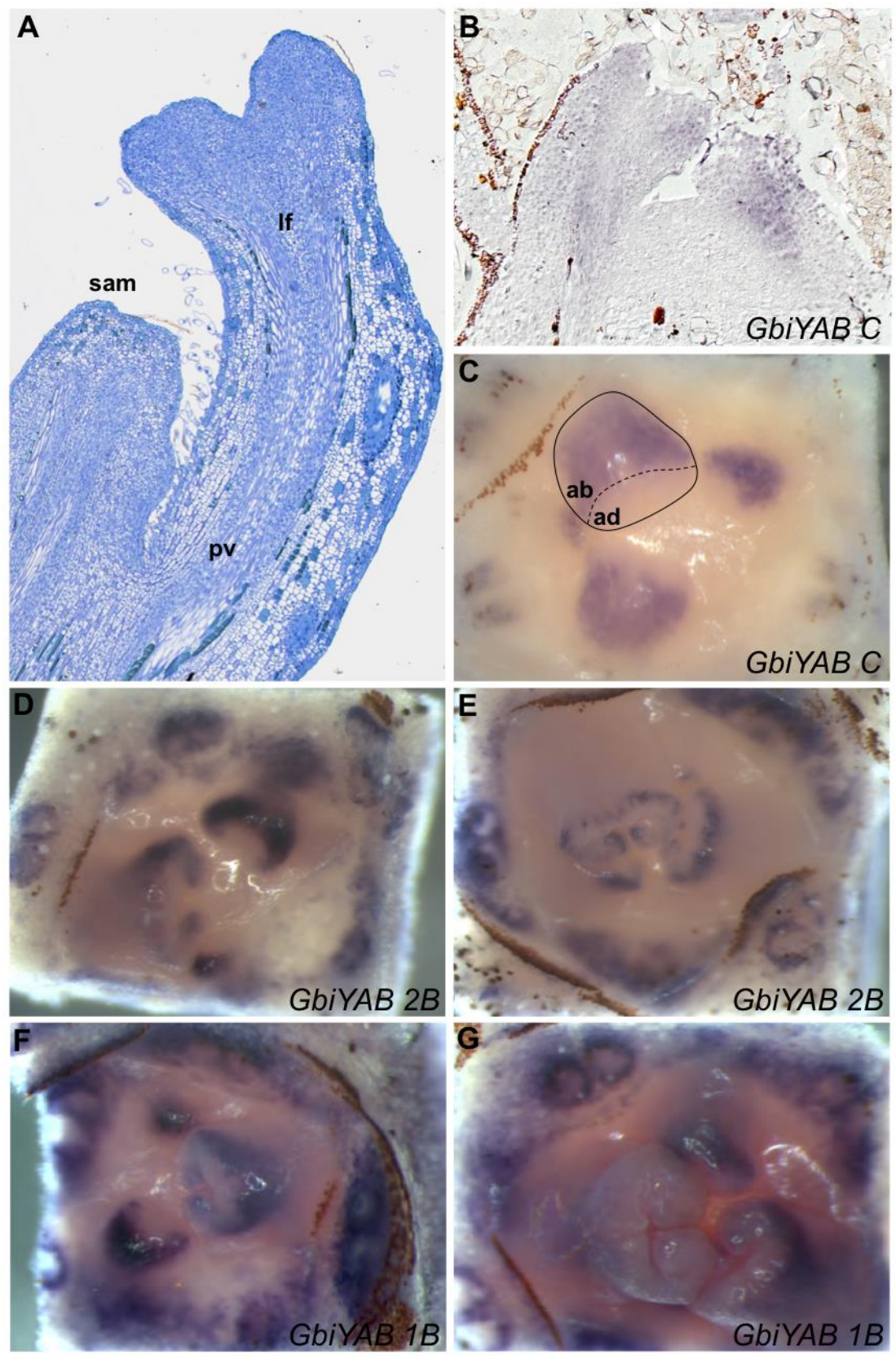

Figure 4 
A

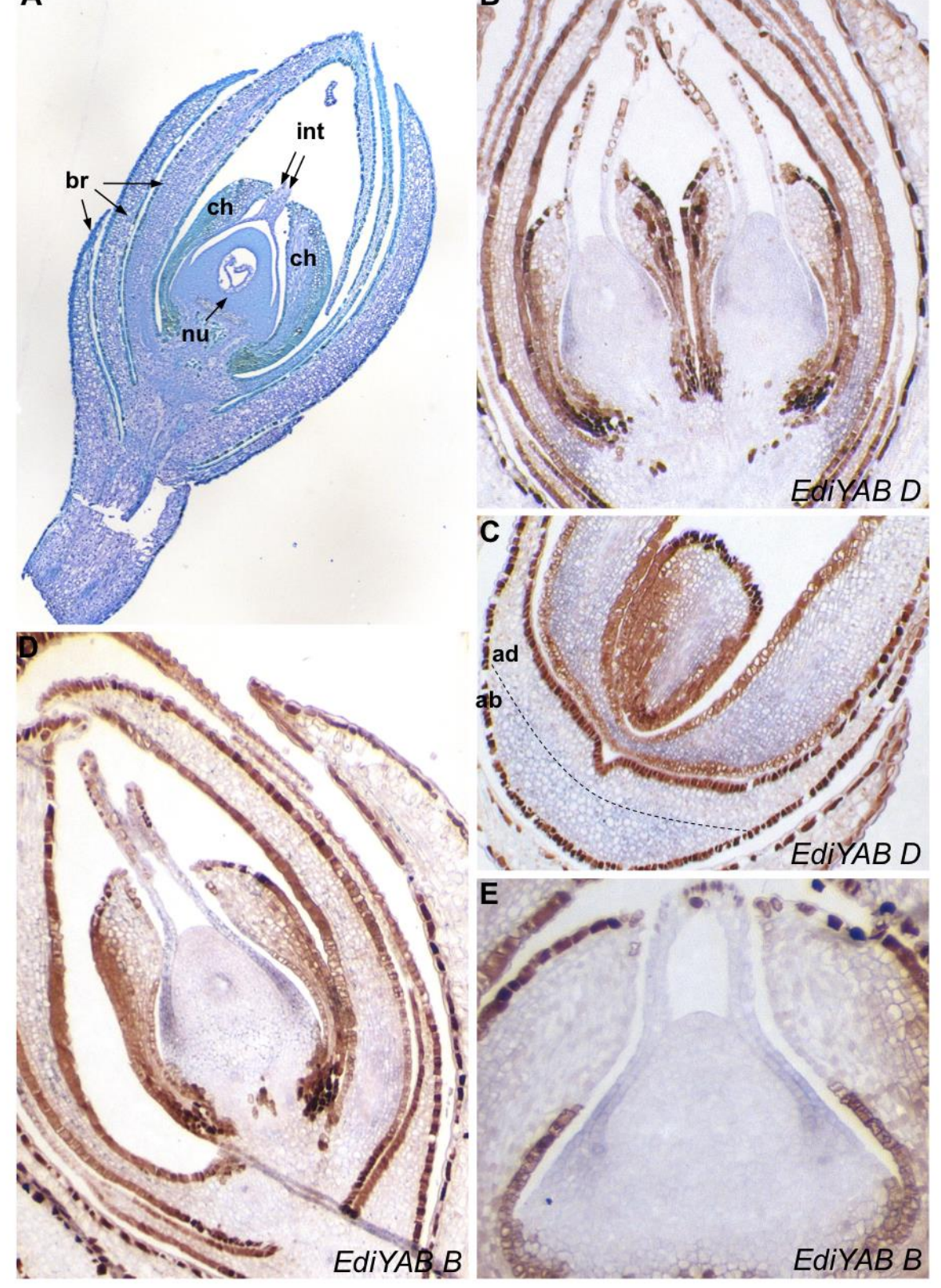

Figure 5 


\section{University Library}

\section{- M M N E R VA A gateway to Melbourne's research publications}

Minerva Access is the Institutional Repository of The University of Melbourne

Author/s:

Finet, C;Floyd, SK;Conway, SJ;Zhong, B;Scutt, CP;Bowmanb, JL

Title:

Evolution of the YABBY gene family in seed plants

Date:

2016-03-01

Citation:

Finet, C., Floyd, S. K., Conway, S. J., Zhong, B., Scutt, C. P. \& Bowmanb, J. L. (2016).

Evolution of the YABBY gene family in seed plants. EVOLUTION \& DEVELOPMENT, 18 (2), pp.116-126. https://doi.org/10.1111/ede.12173.

Persistent Link:

http://hdl.handle.net/11343/290818 